\title{
El Psicoanálisis Relacional y la Teoría del Apego ${ }^{1}$
}

\author{
Alejandro Ávila Espada \\ Universidad Complutense, Madrid \\ Instituto de Psicoterapia Relacional, Madrid
}

\begin{abstract}
En sus cincuenta años de existencia, la Teoría del Apego ha seguido su propia evolución como teoría evolutiva con implicaciones clínicas, mientras que el Psicoanálisis, en su plural conjunto de teorías, ha tenido más dificultades para afrontar la revisión de su teoría clásica del desarrollo. En este trabajo se considera el impacto que las primeras investigaciones modernas sobre el desarrollo afectivo y social, y en especial teoría el apego ha tenido en la transformación del psicoanálisis contemporáneo, y más concretamente en la convergencia de propuestas teóricas, técnicas y clínicas que se conocen actualmente como Psicoanálisis Relacional. Finalmente se discute si la teoría del apego puede fundamentar una estrategia de psicoterapia, o es una contribución más, aunque destacada, a la concepción relacional de la psicoterapia contemporánea.

Palabras clave: Investigación del Desarrollo Infantil, Apego, Psicoterapia, Psicoanálisis Relacional
\end{abstract}

In its fifty years of existence, Attachment Theory has followed its own evolution as an developmental theory with clinical implications, while Psychoanalysis, in its plural set of theories, has had more difficulties in facing the revision of its classical development theory. This paper considers the impact that the first modern investigations on affective and social development, and especially attachment theory, has had in the transformation of contemporary psychoanalysis, and more specifically in the convergence of theoretical, technical and clinical proposals that are known currently as Relational Psychoanalysis. Finally, it is discussed whether attachment theory can support a psychotherapy strategy, or is a further contribution, although outstanding, to the relational conception of contemporary psychotherapy.

Key Words: Infant Research, Attachment, Psychotherapy, Relational Psychoanalysis

English Title: Relational Psychoanalysis and Attachment Theory

\section{Cita bibliográfica / Reference citation:}

Ávila Espada, A. (2020). El psicoanálisis relacional y la teoría del apego. Clínica e Investigación Relacional, 14 (1): 45-61. [ISSN 1988-2939] [Recuperado de www.ceir.info ] DOI:

$10.21110 / 19882939.2020 .140102$

\begin{abstract}
${ }^{1}$ Ponencia presentada en la VIII Reunión Bienal de IARPP-España, Sevilla, 18 y 19 Octubre de 2019, como discusión a la exposición de Mario Marrone "La Teoría del Apego y el Proceso Terapéutico". Fragmentos de este trabajo fueron incluidos en la conferencia dictada en las VII Jornadas Científicas "APEGO, INVESTIGACIÓN Y PSICOTERAPIA" celebradas en Valencia, 15 de Febrero de 2014, organizadas por el Instituto de Psicoterapia de Orientación Psicoanalítica y Antropología (IPSA-Levante) y el Col-legi Oficial de Psicòlegs de la C. Valenciana ${ }^{2}$ Alejandro Ávila Espada, Ph.D., Psicólogo Clínico. Catedrático de Personalidad, Evaluación y Tratamientos Psicológicos, Universidad Complutense (y anteriormente en la Universidad de Salamanca) desde 1990. Presidente de Honor del Instituto de Psicoterapia Relacional (Madrid), y miembro de la International Association of Psychoanalytic Self Psychology (IAPSP). Miembro de Honor de la Federación Española de Asociaciones de Psicoterapeutas (FEAP) de la que fue primer presidente. Miembro del Board of Directors de la International Association for Relational Psychoanalysis and Psychotherapy (IARPP) en el periodo 2011-2018. Editor de la Revista electrónica Clínica e Investigación Relacional. Entre sus obras: Manual de Técnicas de Psicoterapia. Un enfoque psicoanalítico (Madrid: Siglo XXI, 1994); Investigación en Psicoterapia. La contribución psicoanalítica (Barcelona: Paidos, 1998), ambos con J. Poch; Introducción a los tratamientos psicodinámicos (con B. Rojí y L.A. Saul; Madrid: UNED, 2004); La tradición interpersonal. Perspectiva social y cultural del psicoanálisis (Madrid: Ágora Relacional, 2013) y de Relational Horizons: Mediterranean voices bring passion and reason to relational psychoanalysis (Astoria, NY: International Psychoanalytic Books, 2018).
\end{abstract}

CelR Vol. 14 (1) - Abril 2020 ISSN 1988-2939 - www.ceir.info

(c) Derechos reservados/Copyright de Clínica e investigación Relacional y los autores. Prohibida la reproducción total o parcial sin autorización expresa. Este material es para uso científico y profesional exclusivamente y puede contener información clínica sensible. Los editores no se responsabilizan de los contenidos de los autores. Dirigir las consultas sobre derechos y autorizaciones a ceir@psicoterapiarelacional.es 
Cincuenta años después de Attachment and Loss, nos reunimos hoy aquí en Sevilla para reconocer el legado de la obra de Bowlby (1969, 1973, 1980, 1988), y para revisar la riqueza de sus aportaciones, tanto directas como indirectas, a la práctica clínica, que nosotros enfocamos desde los desarrollos del psicoanálisis relacional.

Está suficientemente documentado que una de las principales intenciones de Bowlby con la Teoría del Apego fue forzar el cambio en la teoría psicoanalítica desde su inmovilismo a la hora de mantener una teoría del desarrollo psicosexual especulativa y no basada en la observación, hacia una teoría del desarrollo basada en la observación e investigación científica. Siguiendo la famosa expresión de Fonagy (2001) sobre la "mala sangre" entre ambas disciplinas, el hecho es que, 50 años después ${ }^{1}$, la Teoría del Apego ha seguido su propia evolución como teoría evolutiva con implicaciones clínicas, y el Psicoanálisis, aunque cada vez más plural y abierto, no ha afrontado la revisión en profundidad de su teoría del desarrollo, si bien los esfuerzos por conseguirlo han sido notables por parte de muchos autores (Emde, Fonagy, Eagle...). El planteamiento de Bowlby, aunque original en su esencia, había venido precedido por los que podemos considerar la cultura de los "psicoanalistas precursores de la teoría del apego": Ferenczi (y su concepto de amor pasivo de objeto, 1933), Balint (y su concepto de objeto de amor primario, 1937), Hermann (y su concepto de instinto de adhesión, 1936) o Suttie (con sus instintos adaptados a la infancia, entre ellos el de 'apego simple a la madre', 1935). Incluso las observaciones de Anna Freud o Melanie Klein, cuando describen el comportamiento de los niños con sus madres, constatan lo que después se denominó apego, aunque ellas no modificaron por ello sus teorías, siguiendo la estela del propio Freud, que aunque intuía y apuntaba posibles revisiones de su teoría, no las abordó. Pero las propuestas de Bowlby fueron más allá de estos precedentes, y como subrayan Seligman (2018) y Slavin y Kriegman (1992) Bowlby fue pionero en sostener que el cuidado parental es un requisito esencial para la reproducción de las especies al incluir la motivación social en la perspectiva de la evolución biológica. Esta "brecha" entre la Teoría del Apego y el Psicoanálisis se ha estrechado y difuminado cuando se incorporan los hallazgos de la investigación del desarrollo respecto del papel de la intersubjetividad temprana en la configuración de la base segura que se establece en el vínculo con el cuidador, y más tarde en el despliegue de la relacionalidad del adulto. Ha sido Peter Fonagy (2001) quien más énfasis ha puesto en subrayar las facetas que la Teoría del Apego y el Psicoanálisis tienen en común: la importancia de los cuidados tempranos, el papel de los procesos de mentalización, el papel de las concepciones sobre el sí mismo y sobre los otros como determinantes de la conducta interpersonal del sujeto, el contexto relacional en el que se produce el desarrollo cognitivo, y las motivaciones fundamentales para la vinculación con los otros. 
Para Mitchell (1999, 2000) la Teoría del Apego de Bowlby supuso una confrontación excesivamente directa con la teoría pulsional dominante en el psicoanálisis de su época, y en especial con la teoría freudiana del desarrollo psicosexual. Si los procesos del Apego son relevantes en el desarrollo afectivo del niño, la psicodinamia de las pulsiones queda relegada, a nivel explicativo y evolutivo, a un plano secundario. Son los vínculos -en el plano intersubjetivo y observable- y su papel lo que determina el desarrollo, no las pulsiones y sus representaciones, ni su dinámica inconsciente. Son las reacciones observadas en los niños ante la separación y/o pérdida de sus cuidadores, lo que fundamenta la relevancia del apego y sus variantes adaptativas, que, aunque promueva un mundo representacional característico, este es producto de la calidad del vínculo y sus perturbaciones, con las particulares formas de adaptación al ambiente intersubjetivo (sostenedor o carencial).

Antes de ocuparnos de las relaciones entre las diferentes teorías y observaciones clínicas, hagamos un recorrido breve por la historia de la Teoría del Apego.

\section{El desamparo materno y los procesos de Apego: De Spitz y Harlow a Bowlby²}

La evidencia de que el ser humano entra en relación con el mundo social a través de un sistema de vínculos, en el que el llamado "primer vínculo" juega un papel de especial significación, fue asumida sin discusión en la segunda mitad del siglo XX. Proto-configurado en las etapas avanzadas de la vida uterina, donde algunos autores ya señalaban la existencia de patrones elementales de comunicación en los que lo biológico es el organizador, el sistema de vinculación se va estableciendo de manera compleja durante los primeros meses de vida con el cuidador principal o figura materna ${ }^{3}$. Las propiedades de este sistema de vinculación tienen no solo un impacto decisivo en el desarrollo cognitivo, afectivo y social del niño/a, sino que habrá continuidad (en sus aportes y en sus carencias) entre las propiedades de esos primeros vínculos y los que se forman a lo largo de la vida. En la vinculación se configuran y construyen las capacidades del sujeto, que se modulan y expresan a lo largo de todo el ciclo vital.

Los estudios sobre la importancia de la relación precoz entre padres e hijos fueron iniciados por Anna Freud y Dorothy Burlingham en la Segunda Guerra Mundial, observando los efectos traumáticos de las alteraciones familiares causadas por la guerra (Freud y Burlingham, 1973). A continuación, René Spitz realizó los primeros estudios, ya clásicos, en que se comparaba el desarrollo de niños criados en un orfanato para niños abandonados, cuidados por enfermeras que mantenían escaso contacto con los niños, en un entorno sin apenas contacto visual con su entorno (privación sensorial y social), con el de otros niños criados en una guardería de una prisión para mujeres, que eran cuidados por sus madres, en un entorno abierto a la comunicación social (Spitz, 1945, 1946; Spitz y Wolf, 1947). Al final del primer año de vida, los niños del orfanato 
presentaban síndromes que se describieron como "hospitalismo" (o "depresión anaclítica" en su denominación posterior), inhibición, escasa alegría, eran muy susceptibles a las infecciones, y en el segundo y tercer año mostraban retraso del desarrollo en casi todos los planos, mientras que los niños de la guardería mostraban parámetros similares a los criados en familia. Aunque los estudios de Spitz tenían muchas deficiencias metodológicas, sus resultados se han venido confirmando en líneas generales (Dennis, 1960; Bowlby, 1975).

Tras Spitz, el siguiente descubrimiento relevante se produjo de forma accidental. Margaret y Harry Harlow, dos psicólogos de la U. de Wisconsin que preparaban monos para experimentos, descubrieron los potentes efectos psicológicos y sociales de 6 meses de aislamiento total en la crianza de los monos, comprobando la existencia de un período crítico de desarrollo social. En estudios posteriores (Harlow, 1958; Harlow y cols., 1965), descubrieron que si al mono aislado se le proporcionaba un maniquí de alambre cubierto con felpa que simulaba el pelo de la madre, manifestaba un comportamiento de apego que permitía la aparición de una conducta social normal, y si además de la presencia de la "madre sustituta" se le permitía el contacto diario, durante unas horas, con un compañero que pasaba el resto del día con los monos de su colonia. Décadas más tarde, un nuevo experimento (Suomi y Harlow, 1975) prueba que el síndrome puede revertirse por completo con la ayuda de monos con determinados rasgos caracterológicos (obstinación y agresividad para insistir en la interacción con el mono aislado) que ejercen como "psicoterapeutas", de forma que los monos deprivados lograban, tras 6 meses de "interacción terapéutica", recuperar la salud y casi desaparecían los signos de desamparo. De manera homóloga se ha investigado también repetidamente el impacto de la privación sensorial y social en la organización neural y cortical.

Esta línea de trabajo fue notablemente ampliada por John Bowlby $(1960,1976)$ quien formuló la hipótesis de que el niño indefenso mantiene la proximidad de su cuidador por medio de un sistema de patrones de respuestas emocionales y comportamentales que denomina "sistema de apego". Para Bowlby, este sistema es innato, instintivo, motivacional, como necesidad básica que organiza la conducta del bebé para buscar la proximidad y comunicación con su madre4. Este patrón evolutivo incrementa las posibilidades de supervivencia del bebé, permitiendo al cerebro inmaduro usar las funciones maduras de las figuras parentales para organizar sus propios procesos (una idea convergente con la gestación extrauterina, la intersubjetividad temprana y el "exocerebro" propuesto por Bartra), un conjunto de experiencias de sintonía, comunicación y acción que señala Kandel se codifican en la memoria procedimental (la única disponible en esa temprana etapa) como expectativas que ayudan al niño a sentirse seguro.

Bowlby describe las respuestas de apego y de separación, y divide esta última en dos fases: protesta y desesperación. Los acontecimientos que obstaculizan la proximidad del niño al objeto 
de apego provocan protesta, apego, seguimiento, búsqueda, llanto y activación fisiológica aguda que dura de minutos a horas. Las conductas de protesta pueden restablecer la proximidad, y si se recupera el contacto, desaparecen las conductas de apego mediante un mecanismo de retroalimentación, y se activan otras conductas, principalmente la exploratoria. Pero si la separación es prolongada, la desesperación y la tristeza sustituyen a la ansiedad y la ira. Esta desesperación "prepara" al niño para una supervivencia pasiva prolongada, conservando energía y alejándose del peligro. Estos patrones de apego temprano han sido también observados experimentalmente, además de en humanos y primates, en roedores.

A partir de los trabajos de Bowlby, Mary Ainsworth desarrolló un procedimiento para clasificar la cualidad del apego de un niño hacia un cuidador entre los 12 y los 18 meses de edad, el procedimiento de la Situación Extraña. Este procedimiento de laboratorio induce un estrés leve en el niño/a incluyendo dos separaciones breves del cuidador y dos reuniones con él. En los primeros estudios de Ainsworth, que han sido consistentemente replicados, la sensibilidad de la madre a las comunicaciones del infante en el hogar predecía una estrategia de apego seguro por parte del infante en el laboratorio. Por el contrario, el ligero rechazo materno al contacto íntimo en el hogar predecía una estrategia de apego evitador, y la inconsistencia materna en respuesta a las señales del infante predecía una estrategia de apego ambivalente (Main, 2000). Surge así la primera clasificación de los patrones de apego con tres tipos: Seguro (B); Inseguro/evitativo (A); Inseguro/Resistente-Ambivalente (C).

Aunque Bowlby resaltaba la continuidad entre las conductas de apego humano de seguimiento, llanto y aferramiento y conductas de apego similares en otros primates, Lyons-Ruth (2006) sostiene, siguiendo a Tomasello (2007) y Hobson (2002) que la organización del apego humano es radicalmente diferente de la organización del apego en todas las demás especies. Esto es debido a las capacidades únicas del infante humano para el intercambio intersubjetivo por su naturaleza cooperativa.

Los planteamientos conceptuales y de investigación de Bowlby se perfilaron "a pesar de" los modelos psicoanalíticos imperantes en su época, y al abrirse paso dejaron fecundas cuestiones para los modelos psicoanalíticos posteriores. Buena parte de las teorías kleinianas se vieron superadas por las evidencias de la investigación del desarrollo, incluida la teoría del apego. Las intuiciones de Winnicott (1960) a partir de su clínica ya habían puesto la atención sobre como ciertas variaciones sutiles dentro de un "ambiente esperable promedio" podían producir diferencias individuales en el funcionamiento cognitivo y social del infante. Igualmente Bowlby resaltó (1973) la existencia de modelos representacionales incompatibles en el infante, en el que a nivel consciente pueden reflejar las comunicaciones parentales explícitas, mientras que a nivel inconsciente refleja lo que el niño/a realmente experiencia con ellos. Las defensas y fantasías que 
el infante construye derivan de las experiencias con los cuidadores, no de la dinámica pulsional, y este es un ámbito en que la investigación del apego a través de la identificación de las consecuencias interpersonales de los diferentes patrones de apego ha contribuido a una mejor comprensión actual de los mecanismos de defensa. Fue crucial que la teoría del apego constatase que las fantasías se desarrollan a partir de las experiencias actuales en la realidad intersubjetiva, y como las representaciones inconscientes y las creencias recogen equivalencias simbólicas de la influencia real de las figuras parentales (tanto la estructurante y adaptada a las necesidades plurales de infante; como la dañina o carencial ante las mismas) (Eagle, 1987). Supone la constatación de que los sucesos y relaciones actuales determinan los procesos de organización de la subjetividad del infante -superados ya los planteamientos pulsionales o endogenistas de S. Freud o M. Klein-y que la subjetividad del infante evolucionará y se construirá entre la orientación a la gratificación relacional de sus necesidades o la adaptación patógena a las necesidades de sus cuidadores, todo ello inscrito en su matriz relacional.

Fairbairn (1952) fue claro en su planteamiento de que los modos tempranos de relacionarse, expresados en la búsqueda de objeto relacional por parte del infante, es el vector principal de estructuración de la subjetividad. Más tarde fue Kohut (1984) quien planteó el papel del objeto relacional en la integración del self, operando este a través de la provisión de una base segura en la función de objeto del self. Kohut nos dejó como legado indagar más sobre las relaciones entre el apego inseguro y la falta de cohesión del self, una cohesión necesaria para el desarrollo integrado y saludable del Sí mismo, y que clínicamente observamos en los apegos inseguros persistentes a objetos relacionales con los que se mantiene la expectativa de provisión. Una observación subrayada por Mitchell (1988) para quien el vínculo de apego a un objeto "malo" puede preservar la continuidad de la matriz relacional necesaria para la configuración de la subjetividad. Permanece como reto de la investigación y de la clínica contemporánea explorar las convergencias (y divergencias) entre la teoría del apego y la psicología del self.

Recapitulando, ¿necesitamos "encajar" los conceptos teóricos o derivados de la observación que proceden de los "modelos psicoanalíticos" con los de la "teoría del apego"? La "búsqueda de objeto" de Fairbairn, el "Amor de objeto primario" de Balint, la "Matriz relacional" de Mitchell, ¿tienen que ser absorbidos por o integrados con los conceptos de la teoría del Apego? Nos parece un forzamiento innecesario; unos y otros conceptos han de flexibilizarse para incorporar los matices que aportan los demás, y todos ellos pueden clarificar nuestra práctica.

\section{Apego y Psicopatología}

Los nexos claros entre las estrategias de apego infantil y la psicopatología no fueron apreciados hasta que Main y Solomon (1990) describieron las formas desorganizadas del apego, 
describiendo un cuarto patrón (D) (Desorganizado/Desorientado). Desde entonces se han acumulado las pruebas que apoyan la relación entre las estrategias de apego desorganizado en la infancia y la internalización y externalización de problemas de comportamiento durante la etapa preescolar y los primeros años de escolarización, poniéndose de manifiesto que las estrategias desorganizadas en la infancia a menudo se reorganizan durante la etapa preescolar en una gama de estrategias para controlar a las figuras parentales, bien mediante conductas de cuidado ( $p$. ej. organizando, dirigiendo o entreteniendo) bien mediante conductas punitivas o coercitivas hacia los padres. Lyons-Ruth (2006) subraya que, bajo condiciones normales, una relación de apego con un funcionamiento normal, en la cual el infante pueda señalar abiertamente el malestar y recibir una respuesta sensible por parte del cuidador, servirá para amortiguar el choque del infante (y del adulto) contra niveles extremos de la excitación atemorizante. Sin embargo, el sistema de apego puede funcionar mal. Basado en numerosos hallazgos de investigación, el punto de vista dominante actual considera que las formas desorganizadas y controladoras de la conducta de apego representan un mal funcionamiento del sistema relacional de apego en la infancia que expone al infante a un estrés excesivo no modulado.

Entre los hallazgos específicos acerca de los orígenes del apego desorganizado en la comunicación madre-cuidador/bebé, observados a los 4 meses de edad (comparando las futuras díadas seguras y desorganizadas), están los siguientes (Beebe y Lachmann, 2014):

1) Los futuros niños/as con apego desorganizado pueden no sentirse consistentemente vistos por sus madres

2) Experiencian estados de sobre-excitación sin reparación, evocando la desesperación de la madre que se percibe incapaz de atender a su niño/a.

3) Sienten más afectos discrepantes (conflicto, confusión, pelea) y también sus madres

4) Se sienten menos seguros en la predicción del siguiente estado emocional, y se desestabilizan

5) Se sienten incapaces de "leer" el estado emocional de la madre (están como ante una pared)

6) Les resulta difícil percibir que sus madres sienten y reconocen su malestar, y las madres tampoco lo perciben (la madre sonríe o se sorprende ante el malestar, la madre desconecta la mirada, no hay juego de conexión significativa de regulación mutua; la madre "no cambia con él") 
7) Los investigadores infieren consistentemente que los bebés no se sienten emocionalmente conectados con sus madres, ni consiguen "conocer" su mente; en consecuencia, tienden a fallar en reconocerse a Sí mismos.

La ya extensa literatura de investigación sobre el apego ha ofrecido una base científica para plantear los factores relacionales y biológicos que contribuyen a muchas formas de psicopatología. Paralelamente, la investigación neurobiológica ha hecho aportaciones relevantes sobre los efectos de la respuesta de provisión materna a los patrones de apego. En particular se ha demostrado que el patrón de apego seguro reduce la respuesta hipotalámicohipofiso-suprarrenal (concentración plasmática de glucocorticoides) a distintos factores de estrés, disminuyendo el miedo y la vulnerabilidad ante una enfermedad relacionada con el estrés. Mientras que cuando se prolonga la separación aumenta significativamente la concentración plasmática de corticotropina (ACTH) y la respuesta de los glucocorticoides al estrés. Los numerosos estudios realizados en este campo muestran que las diferencias (naturales) en la interacción de los niños/as con la figura materna son factores de riesgo cruciales para la futura respuesta del sujeto ante el estrés, y también que la separación prolongada tiene efectos adversos sobre el hipocampo. El estrés repetido (p.e. la exposición a una concentración elevada de glucocorticoides ${ }^{5}$ durante varias semanas) causa atrofia en las neuronas del hipocampo, un proceso reversible si se elimina el estrés o las concentraciones de glucocoticoides, pero si la exposición al estrés (o a la concentración elevada de glucocorticoides) se prolonga meses o años, la afectación es permanente y la atrofia neuronal en el hipocampo puede ser irreversible, causando alteraciones significativas en la memoria (declarativa), y lo que pudiera parecer efecto de la represión sea en realidad una amnesia real (afectación del sistema medial del lóbulo temporal del cerebro). En el área de la memoria, Kandel (1999) señala la enorme importancia que tiene la relación entre los primeros procesos mentales inconscientes y los posteriores procesos conscientes:

"El estrés en las primeras etapas de la vida causado por la separación de la madre produce una reacción en el niño que perdura principalmente en la memoria procedimental, el único sistema de memoria bien diferenciado de que el niño dispone en esa etapa, pero su acción sobre el sistema de memoria procedimental desencadena una serie de cambios que terminan por afectar al hipocampo y, como resultado, alteran permanentemente la memoria declarativa" (Kandel, 1999, pp.88-90)

Sigue en estudio, aunque las evidencias son prometedoras, la investigación acerca de cómo vínculos ulteriores de calidad (p.e. en la psicoterapia) y los cambios en las concentraciones de $\mathrm{CRF}^{6}$ o glucocorticoides, pueden contribuir a detener o invertir las alteraciones neuronales en el hipocampo. 
Concluimos está consideración de los nexos entre el apego y la psicopatología, señalando la conexión que se ha establecido en diversos estudios entre las características del diálogo parentofilial que se asocian con flexibilidad y elasticidad (o su ausencia) y su influencia en el curso del desarrollo posterior infantil. La calidad de la comunicación que predice buenos indicadores de desarrollo incluye la apertura a la gama completa de comunicaciones afectivas (Ainsworth y cols., 1978) así como iniciativas de ambos participantes en un diálogo equilibrado y recíprocamente regulado, donde se llevan a cabo transacciones activas corrigiendo fallos, malentendidos y conflictos de intereses (Tronick, 1989). Este escenario implica que se están dando relaciones interpersonales cargadas con un tono emocional positivo, y que se despliegan modelos procedimentales actuados que son válidos para llevar a cabo relaciones coherentes, integradas, flexibles y abiertas a la nueva información. Lyons-Ruth (2003) resume así las características de la "comunicación coherente" que se relaciona positivamente con el desarrollo (y paralelamente con el cambio psicoterapéutico en el adulto), las cuales completamos con algunas matizaciones:

1. Se requiere una estructuración activa del diálogo con la finalidad de poner de relieve las carencias, necesidades, puntos de vista y preferencias del niño (o adulto), tanto actuales como emergentes: se admite de manera explícita la dificultad y la importancia de conocer la mente del otro.

2. El contenido de la comunicación "cara a cara" es esencial: las expresiones faciales que indican sintonía emocional, comprensión, implicación para el cambio, estar con él en la lucha y aceptación de su experiencia favorecen la integración de las experiencias y el cambio positivo; las que indican alarma, reproche, invalidación, distancia, rechazo, potencian la desorganización patológica. Se trata de comunicación corporeizada sobre la que discurre la oportunidad del cambio compartido, en el que ambos partícipes están implicados (Bucci, 2011; Slavin y Kriegman, 1998)

3. Es decisiva la búsqueda activa de reparación de los malentendidos: se necesita una mutua contribución a la regulación del diálogo y a la reparación de los equívocos.

4. Elevación activa del diálogo a nuevos niveles de conciencia por parte del interlocutor más desarrollado: aparece la paradoja de que la relación esté regulada de manera recíproca a pesar de la asimetría evolutiva.

5. Compromiso y lucha activos junto al niño en los periodos de reorganización y cambio de la percepción propia y de los otros, con atenta re-evaluación del peso de las iniciativas y de la capacidad de dirigir la relación por parte del niño: aparece la paradoja de que las iniciativas de la relación están equilibradas a pesar de la asimetría de poder. 
La investigación sobre el apego ha venido a dejar patente que los encuentros que tienen que ver con el apego y se dan en relaciones de intimidad social son regulados por "modelos internos operativos" o representaciones procedimentales actuadas sobre cómo hacer cosas con otros que se construyen tempranamente, y permanecen disponibles para procesar las experiencias ulteriores. Contribuir a cambiar estos modelos internos operativos será la tarea esencial de la psicoterapia.

\section{Apego e Intersubjetividad, dos procesos paralelos, pero con tiempos y funciones diferenciadas}

¿Es el Apego un sistema motivacional clave para la intersubjetividad? Compartiendo con LyonsRuth (2006) esta pregunta, y volviendo a las propuestas sobre la Intersubjetividad de Daniel N. Stern, Ammanitti-Trentini (2011) destacan que los intercambios intersubjetivos entre madre y bebé son parte de lo que Stern denominó "un sistema de motivación primario e innato, fundamental para la supervivencia de la especie, y tiene un estatus como el sexo o el apego" (Stern, 2004, p. 97). Estos intercambios intersubjetivos se desarrollan desde el nacimiento entre el bebé y la madre, pero también con el padre, creando un contexto triádico interactivo (FivazDepeursinge \& Corboz-Warnery, 1999). Teniendo como base observaciones neurobiológicas, puede suponerse que el sistema de ser padres está unido al sistema básico intersubjetivo de motivación, es una extensión del mismo, y es esencial para su reproducción a través de generaciones. La entrada en la maternidad provoca profundos cambios psicológicos en una mujer durante el embarazo y después del nacimiento del bebé. Hay normalmente un cambio básico en el sentido general del self para incluir la identidad maternal, incluyendo la activación de una configuración psíquica concreta, específica de la maternidad, una "constelación de maternidad" (Stern, 1997). La investigación en torno a los circuitos maternales neurobiológicos ha revelado que regiones específicas del cerebro son activadas cuando diferentes sistemas motivacionales implicados en las funciones parentales se observan en el comportamiento de la madre (Lichtenberg, 1989; Nitschke y col., 2004, Schore, 2003).

Frente a quienes consideran que el sistema de apego y el sistema intersubjetivo puedan funcionar como sistemas motivacionales por separado, Lyons-Ruth (2006) defiende, con Tomasello (1999) y Hobson (2002) que la intersubjetividad es una condición de lo humano, una función esencial de la mente. No es posible inhibir o desactivar voluntariamente el desarrollo y el funcionamiento de nuestra conciencia de otras mentes de la misma manera que no podemos inhibir voluntariamente nuestra capacidad de abstracción, y en consecuencia no se puede aceptar que la intersubjetividad sea un sistema motivacional encaminado a un objetivo que se activa bajo ciertas condiciones y se desactiva bajo ciertas otras. El cerebro humano no puede desarrollarse y sostenerse sin relacionalidad, la cual es una condición continuamente 
activa de la vida mental. La pregnancia de lo cultural, intersubjetivo en su origen, nos puede conducir con el tiempo a dejar de focalizar conscientemente nuestra atención en los estados mentales de los otros, pero el flujo intersubjetivo básico de leer los estados de los otros y compartir aspectos de nuestras vidas mentales mediante el intercambio de señales afectivas e intencionales es una condición de nuestra existencia y no puede ser ni activada ni desactivada. Nuestra capacidad de cuidar y participar en las vidas emocionales de los otros es una "característica de diseño" del ser humano (Hobson, 2002)7.

Subraya Lyons-Ruth (2006) que las limitaciones de la teoría de Freud no son tan evidentes en ningún otro sitio como en su teoría motivacional. Los mecanismos más flexibles y adaptativos de compartir estados mentales con otros reformulan nuestra herencia evolutiva biológica más determinista. Esta reformulación requiere un cambio radical en nuestras teorías del funcionamiento humano para dar lugar a una visión más clara de cómo el compartir estados mentales a partir del momento del nacimiento cataliza los procesos de aprendizaje socialmente distribuidos de modo que cada generación se apoya sobre el legado de la generación anterior. Al igual que esta capacidad para el compartir intersubjetivo re-contextualiza y subordina el principio de evolución biológica de Darwin aplicado a la evolución humana, también recontextualiza y subordina los sistemas motivacionales humanos de base biológica, incluidos los de Freud, a un contexto de imperativos para la conducta humana más flexible y creado socialmente, en línea con lo que ha planteado Mitchell (1997) en su propuesta para explicar el cambio como resultante de la agencia y la relacionalidad.

Cuando la teoría del apego pasa a interesarse por las representaciones mentales (la llamada tercera fase de la teoría del apego) y a través del estudio del comportamiento de los adultos mediante la AAI, identificando tres patrones (Seguro/autónomo; Rechazante; Preocupado) se destaca como el sentido de seguridad personal alcanzado por el adulto correlaciona más claramente con la habilidad para reflexionar de manera coherente sobre las propias experiencias y recuerdos que con los sucesos actuales. También se acumula evidencia sobre la transmisión intergeneracional de la seguridad personal de los cuidadores a los infantes. Este proceso es equivalente al impacto de los terapeutas -y del vínculo terapéutico que se establece- en los pacientes, una faceta muy relevante del proceso terapéutico donde el conocimiento de los propios terapeutas sobre su estilo de apego incrementa la comprensión de sus propias reacciones emocionales en el encuentro con los pacientes, y en la transmisión que se hace mediante la relacionalidad inherente al proceso terapéutico, donde emoción y reflexión caminan juntas en la evolución de las calidades de la mentalización relacional, de la metacognición y de la construcción constante de un self subjetivo, un proceso compartido y co-determinado por paciente y terapeuta, donde se vive la propia experiencia de despliegue y mejora de la función 
reflexiva, donde sentirse seguro y sentirse comprendido es parte del mismo proceso (Seligman, 2018).

Si examinamos más en detalle el papel de la mentalización, siguiendo a Eagle (2013), este proceso de naturaleza procedimental, está presente ya muy tempranamente, al año de edad (Gergely y Unoka, 2008), aunque en esos momentos tempranos no haya aún función reflexiva y la atribución intencional sea proyectiva. La mejora cualitativa de las capacidades de mentalización, mediante el despliegue de la función reflexiva y su mayor sofisticación, es uno de los ejes evolutivos más relevantes, y a la vez un objetivo terapéutico compartido por la $\mathrm{MBT}^{8} \mathrm{y}$ la $\mathrm{ABT}^{9}$. Más como hecho clínico -y eje del proceso terapéutico- que como propuesta teórica, la función de la interpretación en el psicoanálisis no es tanto el "descubrimiento" de las fantasías y los deseos reprimidos, como despertar la curiosidad de la persona y ampliar su comprensión sobre cómo funciona su mente en relación consigo y con los demás. La mejora cualitativa de la función reflexiva facilita entonces la regulación de la experiencia de los afectos e interviene en la regulación de la activación de patrones cognitivos y relacionales "automáticos", procesos ambos inter-determinados.

Cortina y Liotti (2010) han mostrado que, si bien el apego y la intersubjetividad están íntimamente relacionados a lo largo del desarrollo, pueden ser distinguidos tanto en base a criterios evolucionistas como del desarrollo. Para estos autores la estrategia evolutiva más importante que impulsó la evolución humana fue la aparición de grupos cooperativos que pudieron compartir recursos en igualdad de condiciones $y$, por lo tanto, compitieron favorablemente con otros grupos (selección grupal). Esta estrategia evolutiva muy probablemente evolucionó a través del despliegue de estrategias cooperativas para la crianza y las historias de vida compartida, como Hrdy (2009) ha argumentado ampliamente. La aparición de una forma hiper-cooperativa de sociabilidad requirió una comunicación más efectiva entre los miembros del grupo. Una mejor comprensión de las intenciones y motivos de otros miembros ayudó en la planificación cooperativa conjunta de las actividades de caza y recolección entre los grupos nómadas durante el curso de la evolución humana. En ese contexto, la comprensión de las intenciones, los motivos y los estados mentales de los demás tiene lugar en el nivel intersubjetivo. Esta rica matriz interpersonal y experiencial proporciona un terreno común fértil sobre el cual emergen las actividades simbólicas y el lenguaje. La intersubjetividad fue esencial para desplegar las herramientas de comunicación social y las acciones cooperativas, en un proceso paralelo, pero diferente del apego, que facilita la sensación de protección y seguridad sobre la que se despliega la apertura a lo social extenso. 


\section{¿Podemos basar nuestra concepción de la psicoterapia en el apego o necesitamos una concepción relacional informada por la investigación?}

Bowlby (1988), Holmes (2001 [2009]), Stern (2007), Wallin (2007 [2012], 2014) y Cortina y Marrone (2018), entre otros, han establecido interesantes propuestas e ilustrado casuística sobre el papel de la teoría del apego a la hora de configurar las direcciones estratégicas de la psicoterapia en diferentes ámbitos clínicos.

Fue John Bowlby quien abrió explícitamente este camino cuando publicó en 1988 su conocido ensayo titulado "Una base segura" en el que se adentra en las aplicaciones clínicas de la teoría del apego y la contribución de esta al enfoque estratégico de la psicoterapia al promover la (re)configuración de una base segura en el vínculo terapéutico para facilitar el despliegue de una subjetividad madura. Este mismo camino fue seguido por Jeremy Holmes, con una obra de similar título y objetivo (2009), en la que se presenta una "terapia breve basada en el apego" $(\mathrm{BABI})$ en la que se integran aportaciones de diferentes tradiciones teóricas a la intervención estratégica de tiempo limitado, en la que la exploración de las características de la base segura, la promoción de la función reflexiva -y de la competencia autobiográfica-, la identificación de los esquemas operativos internos, así como la gestión de la ansiedad de separación juegan un papel destacado, todo ello apuntando hacia la dirección estratégica de modificar los estilos de apego del adulto que acude a la consulta.

David Wallin, profundizando en el camino abierto por Holmes, revisó la evolución de la teoría del apego (2012) y trazó los puentes que sitúan a la psicoterapia psicoanalítica relacional, en su acepción amplia, como el contexto natural en el que la teoría del apego contribuye a la ayuda clínica: la construcción del self y sus múltiples facetas; el impacto -y el trabajo- con lo "sabido no pensado", la facilitación de la intimidad, el trabajo dirigido a la mejora de las capacidades de mentalización, la comunicación no verbal, lo corporal y lo enactuado, y especialmente la regulación de las huellas de la experiencia traumática.

Estos puentes ya los había dejado enunciados Stephen A. Mitchell $(1999,2000)$ cuando revisó las convergencias y divergencias entre el apego y la intersubjetividad, en especial en su relato clínico del trabajo con su paciente Connie, donde se re-experiencian patrones de apego tempranos y se evoluciona hacia calidades de intersubjetividad más sustentadoras de una conexión emocional profunda con los otros, y un poder estar en contacto consigo misma más pleno y consistente. Daniel N. Stern (2007) hizo un recorrido semejante cuando comparó la observación de los sucesos acaecidos con la gansa Feli y el trabajo clínico con su paciente Anna, en uno de sus trabajos clínicos más claramente descritos, donde los procesos de apego se inscriben en la evolución de la intersubjetividad. 
Como ha señalado recientemente Mauricio Cortina (2018), la teoría del apego ha ejercido una influencia destacada para estructurar las estrategias de intervención temprana con padres, en la terapia de pareja, y en la psicoterapia del adulto, pero esta influencia es específica a la hora de identificar, comprender y cambiar los modelos representacionales de las figuras de apego (y en consecuencia regular facetas de los patrones de apego desplegados, especialmente en el nivel emocional), sin que sustituya al enfoque más amplio y complejo que requieren cualesquiera de esas intervenciones. En su reciente compilación, Cortina y Marrone (2017) trazan los puentes para contextuar el trabajo clínico del apego como parte del conjunto de los sistemas motivacionales que fueron descritos por Lichtenberg, Lachmann y Fosshage (2011) y que han sido también revisados o adaptados de esta teoría por otros autores (p.e. H. Bleichmar, 1997), y configurados a través del trabajo específico de las "constelaciones representacionales" (Marrone).

Más allá del tributo a las aportaciones precedentes, nosotros pensamos el Apego y los demás sistemas motivacionales, desde la perspectiva relacional, nuestra teoría clínica de referencia. La contribución relacional a la clínica se apoya en todo lo que nos ha aportado la investigación del desarrollo humano de las últimas décadas, y específicamente en el papel de la intersubjetividad en la configuración, despliegue y transformación de la subjetividad. Más allá de los múltiples y diferentes procesos que inciden en el desarrollo humano, el clínico no restringe su comprensión ni su estrategia a ninguna teoría, por fecunda que sea, sino que configura su bagaje conceptual y técnico, sea cual sea la cultura teórico-técnica de la que proceda, a través de un conocimiento detallado y experiencial de los desarrollos actuales de la Teoría del Apego, la Teoría de la Mentalización, de la Teoría de la Especificidad del desarrollo y del cambio, y particularmente de la investigación del Proceso de Cambio. Y es responsable de sostener la motivación para actualizarse en los avances en Cognición Social, en Neurociencia y Desarrollo Humano. Todos estos conocimientos, integrados con la experiencia, son el núcleo de la acción del psicoterapeuta relacional.

\section{REFERENCIAS}

Ávila, A. (2016). La construcción intersubjetiva del ser humano: La investigación del desarrollo humano fundamenta una clínica relacional. Texto no publicado de la intervención leída en las Jornadas Internacionales EDAl, en Barcelona, 27 de Mayo 2016.

Balint, M. (1937). Early developmental styles of the Ego: Primary object love. En Primary love and psychoanalytic technique (pp. 234-267). New York: Liveright, 1965.

Beebe, B. y Lachmann, F.M. (Eds.). (2014). The origins of attachment. Infant Research and Adult Treatment. New York: Routledge. 
Bleichmar, H. (1997). Avances en Psicoterapia Psicoanalítica. Hacia una técnica de intervenciones específicas. Barcelona: Paidos.

Bowlby, J. (1976). El vínculo afectivo. Buenos Aires: Paidos [Original: Attachment and loss. Vol.1 Attachment. Londres: Hogarth Press, 1969; Traducción de M. Valcarce]

Bowlby, J. (1980a). La pérdida afectiva. Tristeza y depresión. Barcelona: Paidos [Original: Attachment and loss. Vol.3 Loss. Londres: Hogarth Press, 1980; Traducción de A. Baez]

Bowlby, J. (1980b). La separación afectiva. Barcelona: Paidos [Original: Attachment and loss. Vol.2 Separation. Anxiety and Anger. Londres: Hogarth Press, 1973; Traducción de I. Pardal]

Bowlby, J.B. (1988). Una base segura. Aplicaciones clínicas de la teoría del apego. Barcelona: Paidós [Original del mismo año]

Cortina, M. (2018). Avances clínicos de (la) teoría del vínculo de apego en los últimos 25 años. Aperturas Psicoanalíticas, 59: 1-30.

Cortina, M. y Liotti, G. (2010). Attachment is about safety and protection, Intersubjectivity is about sharing and social understanding. Psychoanalytic Psychology, Vol. 27, No. 4, 410-441

Cortina, M. y Marrone, M. (Comps.) (2017). Apego y Psicoterapia. Un paradigma revolucionario. Madrid: Psimática.

Diamond, N. y Marrone, M. (2003). Attachment and intersubjectivity. London: Whurr Publishers.

Eagle, M. (1987). The psychoanalytic and the cognitive unconscious. En R. Stern (Ed.). Theories of the unconscious and theories of the self (pp. 155-189). Hillsdale, NJ: The Analytic Press.

Eagle, M.N. (2013). Attachment and Psychoanalysis. Theory, Research, and Clinical Implications. New York: The Guilford Press

Ferenczi, S., (1933) Confusión de lengua entre los adultos y el niño: El lenguaje de la ternura y de la pasión. Obras Completas. Psicoanalisis IV. Tr. Fco. J. Aguirre. Ed. Espasa Calpe. Madrid, 1984, pp. 139-149.

Fonagy, P. (2004). Teoría del apego y psicoanálisis. Barcelona: Espaxs [Psychoanalysis and Attachment Theory. London: Karnac, 2001]

Fonagy, P., Gergely, G., Jurist, E. \& Target, M. (2002). Affect Regulation, Mentalization, and the Development of the Self. New York: Other Press.

Gergely, G. y Unoka, Z. (2008). Attachment and mentalization in humans: The development of the affective self. En E.L.Jurist, A. Slade y S. Bergner (Eds). Mind to mind: Infant research, neuroscience and psychoanalysis (pp.50-67). New York: Other Press.

Hermann, J. (1936). Clinging and going-in-search. Psychoanalytic Quarterly, 45: 5-36.

Hobson, P (2002). The Cradle of Thought: Exploring the Origins of Thinking. London, MacMillan.

Holmes, J. (1993). John Bowlby and Attachment Theory. London: Routledge

Holmes, J. (2009). Teoría del Apego y Psicoterapia. En busca de una base segura. Bilbao: Desclée de Brouwer [Original de 2001] 
Hrdy, B.S. (2009). Mothers and others: The evolutionary origins of mutual understanding. Cambridge, MA: The Belknap Press.

Lichtenberg, J.D., Lachmann, F.M. \& Fosshage, J.L. (2011). Psychoanalysis and Motivational Systems. A New Look. New York: Routledge.

Lichtenberg, J.D. (2016). A Developmentalist's Approach to Research, Theory, and Therapy. The selected Works of Joseph D. Lichtenberg. New York: Routledge.

Lyons-Ruth, K. (2006). The interface between attachment and intersubjectivity: Perspective from the longitudinal study of disorganized attachment" Psychoanalytic Inquiry, 26 (4): 595-616. [Versión castellana en Aperturas Psicoanalíticas, 2008, n² 29]

Marrone, M. (2009). La teoría del apego. Un enfoque actual Madrid: Psimática, ( $2^{\mathrm{a}}$ ed. Rev.).

Mitchell, S. A. (1988). Relational Concepts in Psychoanalysis: An Integration. Cambridge, MA: Harvard University Press [V. castellana: Conceptos relacionales en psicoanálisis, Mexico: Siglo XXI]

Mitchell, S.A. (1999). Attachment Theory and the Psychoanalytic Tradition. Reflections in Human Relationality. Psychoanalytic Dialogues, 9(1):85-107.

Mitchell, S.A. (2000). Relationality. From Attachment to Intersubjectivity. Relational Perspective Book Series. Volume 20. The Analytic Press, Inc., Hillsdale, New Jersey. [Reseña en castellano de N. Levinton: Aperturas Psicoanalíticas, Revista de Psicoanálisis, Abril 2001, nº 9]

Seligman, S. (2018). Relationships in Development. Infancy, Intersubjectivity, and Attachment. New York: Routledge.

Slavin, M. y Kriegman, D. (1992). The adaptive design of the human psyche: Psychoanalysis, evolutionary biology, and the Therapeutic process. New York: Guilford

Stern, D.N. (2007). A Felicitous meeting of Attachment and Relational Psychotherapy. ATTACHMENT: New Directions in Psychotherapy and Relational Psychoanalysis, Vol. 1, March 2007: pp. 1-7.

Suttie, I.D. (1935). The origins of love and hate. London: Free Association Books, 1988.

Tomasello, M. (2007). Los orígenes culturales de la cognición humana. Buenos Aires: Amorrortu [Original de 1999: The Cultural Origins of Human Cognition. Cambridge, MA: Harvard University Press]

Tomasello, M. y cols. (2010). ¿Por qué cooperamos?. Buenos Aires: Katz [Original de 2009]

Wallin, D.J. (2012). El Apego en psicoterapia. Bilbao: Desclée de Brouwer [Original de 2007].

Wallin, D.J. (2014). Because Connection Takes Two: The Analyst's Psychology in Treating the "Connection-Resistant" Patient. International Journal of Psychoanalytic Self Psychology, 9: 200207.

Winnicott, D.W. (1960). La distorsión del yo en términos de self verdadero y falso. En Los procesos de maduración y el ambiente facilitador (pp. 182-199) Barcelona: Paidos, 1992.

Original recibido con fecha: 19/10/2019 Revisado: 28/02/2020 Aceptado: 15/03/2020 
NOTAS:

${ }^{1}$ El primer volumen de Bowlby de la serie Attachment and Loss fue publicado en 1969.

${ }^{2}$ Partes de este epígrafe fueron redactados para la Conferencia inaugural titulada "Los orígenes del apego en la experiencia relacional temprana mediante la comunicación infante-cuidador y sus implicaciones para la psicoterapia del adulto" en las VII Jornadas Científicas "APEGO, INVESTIGACIÓN Y PSICOTERAPIA" celebradas en Valencia, 15 de Febrero de 2014, organizadas por el Instituto de Psicoterapia de Orientación Psicoanalítica y Antropología (IPSA-Levante) y el Col-legi Oficial de Psicòlegs de la Comunitat Valenciana.

3 Hablamos de "figura materna" como la figura que está más en contacto continuo con el bebé, y con la que se configuran los procesos que serán descritos como fenómenos de la intersubjetividad temprana. Este continuo contacto es tanto el soporte que da satisfacción a las necesidades primarias, protege de los peligros del ambiente, y mantiene una lazo comunicacional que se va diferenciando, complejizando e integrando progresivamente. Hacia los seis u ocho meses (como muy tarde) los bebés normales discriminan la figura materna de otras figuras, habiéndose ya configurado el vínculo de apego, con unas determinadas calidades/propiedades.

${ }^{4}$ La posición de Bowlby y demás teóricos del apego, difiere de la que sostuvieron Margaret Mahler, Pine y Bergman (1975), dentro de las posiciones psicoanalíticas. Holmes (1993) y Fonagy (2004) subrayan que, para Mahler, el desarrollo del niño consiste en una diferenciación progresiva respecto de la madre, como un vector inherente a su naturaleza, un proceso de diferenciación entre yo y no yo, mientras que los teóricos del apego consideran que tanto el bebé como la madre se buscan el uno al otro para relacionarse. Mahler sostiene que el niño se desarrolla a partir de un autismo normal hasta llegar a la individuación Las observaciones de Margaret Mahler han tenido una importante repercusión en la clínica de adultos, ya que han permitido una mejor comprensión de algunos aspectos de la patología grave. Mahler y cols (1975) observaron que algunas madres, en el reencuentro con el bebé, respondían bien retirándose o con agresión, y que la respuesta de los niños era similar a la de los pacientes límites. Para Mahler es importante ver cómo el niño toma conciencia de sus límites corporales, y tiene muy en cuenta el papel que desempeña el cuidador en el desarrollo del self corporal.

${ }^{5}$ Hay dos tipos de receptores de glucocorticoides: los de tipo 1 (mineral-corticoides) y los de tipo 2 (glucocorticoides), ambos presentes en el hipocampo.

${ }^{6}$ CRF: Corticoliberina, la hormona que libera el hipotálamo para iniciar la respuesta hipotalámo-hipofisosuprarrenal.

${ }^{7}$ Aunque se pierda esa prioridad parcialmente en etapas posteriores, lo cual puede ser reforzado por una cultura "individualista" o solipsista

${ }^{8}$ MBT: Terapia basada en la Mentalización

${ }_{9}$ ABT: Terapia basada en el Apego 